\title{
The Test-Retest Reliability of Supraspinatus Cross-Sectional Area Measurement by Sonography
}

\author{
Yang Soo Kim, Nam Yeon Heo, Min Wook Kim
}

Department of Rehabilitation Medicine, Incheon St. Mary's Hospital, The Catholic University of Korea, Incheon 403-720, Korea

Objective To evaluate the test-retest reliability of supraspinatus cross-sectional area measurement by ultrasonography.

Method Both shoulders of 11 normal subjects (22 shoulders in total) were included in this study. The supraspinatus muscle was examined with the arm alongside the body in the coronal oblique and sagittal oblique planes. The occupational ratio of the supraspinatus fossa was measured. To calculate the occupational ratio, the Y view of MRI was reproduced with sonography by locating the suprascapular notch in the coronal oblique plane (in the plane of the scapula) and then rotating the transducer $90^{\circ}$ to that plane. The cross-sectional area was measured using the tracing and ellipse tool. The second measurement was performed 7 days after the initial measurement.

Results The Pearson correlation coefficient and intraclass correlation coefficient between the first and the second occupational ratio measurements were 0.43 and 0.44 , respectively, for the tracing method, and 0.53 and 0.47 , respectively, for the ellipsoidal method. The difference between the first and second occupational ratio measurement was $4.1 \pm 3.9 \%(0.1-13.2 \%)$ for the tracing method, and $4.5 \pm 3.4 \%$ (0.01-10.5\%) for the ellipsoidal method. The maximum difference was $13.2 \%$. The occupational ratio was $86.2 \pm 5.3 \%$ (70.6-95.8\%) for the tracing method and $85.0 \pm 5.2 \%$ (69.3-96.1\%) for the ellipsoidal method.

Conclusion Supraspinatus occupational ratio by sonography is a low to moderately reliable intrarater method. However, the maximum difference was not significant. The main reason for its low to moderate reliability was the narrow value range. Therefore, the study method should be re-evaluated in stroke patients and in patients with rotator cuff disease. Knowledge of the anatomy is a prerequisite to attain intrarater reliability.

Key Words Supraspinatus, Ultrasonography, Test-retest reliability

Received July 8, 2010; Accepted April 8, 2011

Corresponding author: Min Wook Kim

Department of Rehabilitation Medicine, Incheon St. Mary's Hospital, The Catholic University of Korea, 665, Bupyeong-6dong, Bupyeong-gu, Incheon 403-720, Korea

Tel: +82-32-280-5858, Fax: +82-32-280-5040, E-mail: minukkim@nate. com

(c) This is an open-access article distributed under the terms of the Creative Commons Attribution Non-Commercial License (http://creativecommons.org/ licenses/by-nc/3.0) which permits unrestricted noncommercial use, distribution, and reproduction in any medium, provided the original work is properly cited. Copyright $\odot 2011$ by Korean Academy of Rehabilitation Medicine

\section{INTRODUCTION}

Supraspinatus atrophy can occur secondary to pathologies of the central and peripheral nervous system, as well as muscular and tendinous pathology. Supraspinatus is an important target muscle for functional electrical stimulation when there is weakness of shoulder muscles in stroke patients. ${ }^{1}$ Rupture of the supraspinatus tendon causes degeneration and atrophy of muscles and this is 
known to influence prognosis after surgery. ${ }^{2}$ Evaluation of the extent of supraspinatus atrophy is expected to confer benefits to patients experiencing pathologies of the central and peripheral nervous system, as well as muscular and tendinous pathology.

Although the standardized imaging method for assessing supraspinatus atrophy has been magnetic resonance imaging (MRI), ${ }^{3}$ advances in ultrasonographic imaging technology have lead to high correlation with MRI in assessing supraspinatus atrophy. ${ }^{4}$ The portion of supraspinatus sectional area in the supraspinatus fossa demonstrated by the Y-view of MRI is suitable for the measurement of atrophy, ${ }^{5}$ and it has also been possible to demonstrate this by using ultrasonography. ${ }^{4}$

However, subjective factors are known to influence ultrasonographic examinations which do not influence MRI, and evaluation with ultrasonography concurrently leads to divergent results. Moreover, ultrasonography cannot penetrate the skeleton. The Y-view of MRI can visualize the most bony surrounded section at the shoulder such as clavicle anteriorly, supraspinatus fossa inferiorly, scapular spine posteriorly, trapezius muscle superiorly. ${ }^{5}$ It is therefore possible for the Y-view not to be examined using ultrasonography.

The purpose of this study was to evaluate the testretest reliability for eliminating subjective factors and reliability of a follow-up study in ultrasonographic examination, and to verify the relationship between the tracing and ellipsoidal methods. In addition, this study investigated the practical availability of assessing the occupational ratio of supraspinatus fossa in Y-view using ultrasonography.
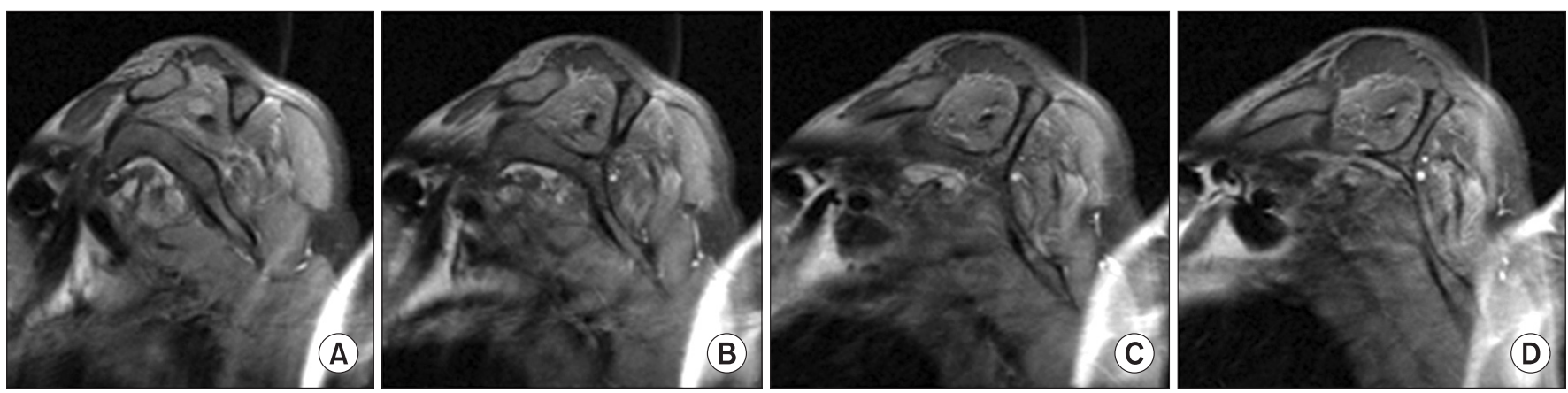

Fig. 1. MRI Y-view (B or C) could be obtained by just one or two images advancing from the lateral (A) to medial (D). The defects of the Y-view were caused by the spinoglenoid notch at A and by the supraspinatus notch at D. 


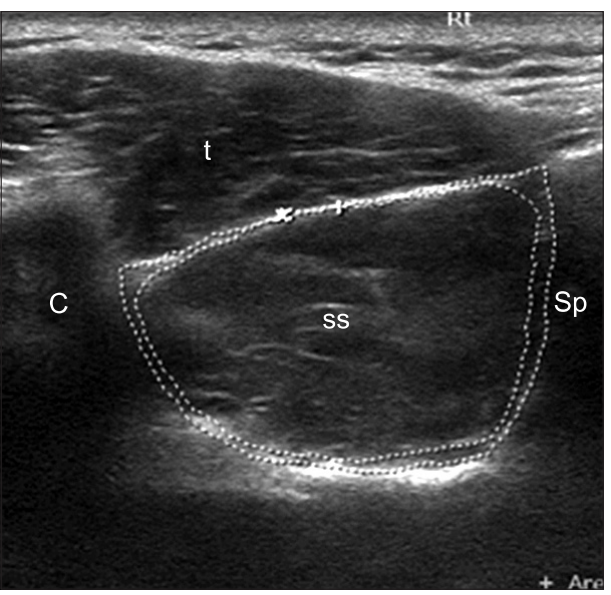

Tracing method

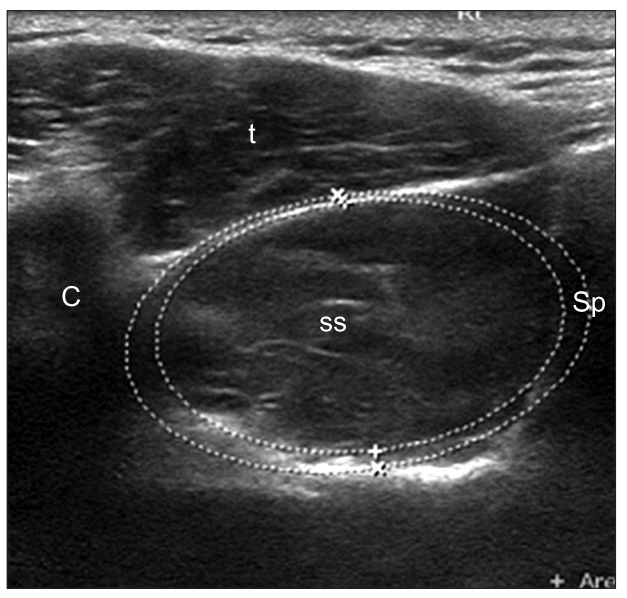

Ellipsoidal method
Fig. 2. Calculation of the occupation ratio by sonography reproducing MRI Y-view. C: clavicle; Sp: Scapular spine; t: trapezius; ss: supraspinatus. of the supraspinatus muscle by the supraspinatus fossa area.

Two methods were used to obtain the cross-sectional area. First, the tracing method was applied, which tracked lines with boundary. Second, was the ellipse tool method, which established an ellipse as a boundary. For the tracing method, the cross sectional area of the supraspinatus muscle was measured at the closest boundary of the muscle. The borders that defined the portion of supraspinatus fossa was set by the internal bony boundary of the $\mathrm{Y}$ view, consisting of the clavicle anteriorly, the cortex of the supraspinatus fossa inferiorly, the scapular spine posteriorly and the inferior border of the trapezius superiorly. For the ellipse tool method, the ellipse was set at the closest line that included the supraspinatus muscle. The border of the supraspinatus fossa was defined by the closest line, including the supraspinatus fossa through the internal bony boundary of the Y-view (Fig. 2).

Two measurements were performed within 7 days to evaluate test-retest reliability. By using the SPSS 14.0 software (SPSS Inc., Chicago, USA), the test-retest reliability was evaluated by calculating the Pearson correlation coefficient and intraclass correlation coefficient (ICC).

\section{RESULTS}

The occupational ratios of the 11 normal subjects were 0.71-0.96 (mean value of 0.86 ) by the tracing method, and 0.69-0.96 (mean value of 0.85 ) by the ellipsoidal method. The Pearson correlation coefficient of the two method was $0.80(\mathrm{p}<0.05)$.
Table 1. The Occupation Ratio of the Supraspinatus Muscle Using Ultrasound and Intrarater Reliability of the Tracing and Ellipsoidal Method

\begin{tabular}{lcc}
\hline & $\begin{array}{c}\text { Tracing } \\
\text { method }\end{array}$ & $\begin{array}{c}\text { Ellipsoidal } \\
\text { method }\end{array}$ \\
\hline Occupation ratio & $\begin{array}{c}0.86 \pm 0.05 \\
(0.71-0.96)\end{array}$ & $\begin{array}{c}0.85 \pm 0.05 \\
(0.69-0.96)\end{array}$ \\
The difference between & $0.04 \pm 0.04$ & $0.05 \pm 0.03$ \\
measurements & $(0-0.13)$ & $(0-0.11)$ \\
Pearson correlation coefficient & $0.43^{*}$ & $0.53^{*}$ \\
Intraclass correlation & $0.44^{*}$ & $0.47^{*}$ \\
coefficient & & \\
\hline
\end{tabular}

Numbers are mean \pm standard deviation

${ }^{*} \mathrm{p}<0.05$

The difference between the two measurements in the evaluation of test-retest reliability was 0-0.13 (mean value of 0.04) for the tracing method, and 0-0.11 (mean value of 0.05) for the ellipsoidal method. The Pearson correlation coefficient was 0.43 for the tracing method and 0.53 for the ellipsoidal method. ICC of two measurements was 0.44 for the tracing method and 0.47 for the ellipsoidal method (Table 1).

\section{DISCUSSION}

In this study we measured both shoulders of 11 normal subjects (22 shoulders). The occupational ratio of supraspinatus muscle was over 0.71 for the tracing method and over 0.69 for the ellipsoidal method, which is within the normal limits according to Thomazeau's classification. Thomazeau et al. ${ }^{5}$ measured 
the occupational ratio of the supraspinatus muscle in the supraspinatus fossa and defined a range between 0.60 and 1.00 as being representative of normal or mild atrophy, 0.40 and 0.60 as being representative of moderate atrophy and less than 0.40 as being severe atrophy.

Because both the tracing and ellipsoidal methods were highly correlated $(\mathrm{r}=0.80)$, it is suggestive that the ellipsoidal method can be used clinically as much as the tracing method. However, the tracing method remains a standard that demonstrates the severity of supraspinatus atrophy more clearly. Further studies are needed for determining the significance of measuring crosssectional areas of the supraspinatus muscle in patients with stroke, rotator-cuff tear and shoulder pain.

The test-retest reliability of ultrasonography was relatively low. ${ }^{6}$ A major reason may be narrow range of measurement. Mean values of the two methods were $0.85(\mathrm{SD}=0.05)$ for the tracing method and $0.85(\mathrm{SD}=0.05)$ for the ellipsoidal method. If the values were distributed from low to high range, the correlation coefficient would be high. However, this study assessed only normal adult males, therefore the measured values were distributed within a similar range, and this resulted in a relatively low correlation coefficient. The maximal difference between the ratios was not high. The mean difference was 0.04 in the tracing method and 0.05 in the ellipsoidal method. The maximum difference was 0.13 . Although the testretest reliability was low, further studies including stroke patients and those with supraspinatus muscle lesions are required.

T1 enhancing image in MRI is the standard method to estimate supraspinatus muscle atrophy, and the sectional area of supraspinatus muscle can be obtained from the Y-view passing through the medial border of the coracoids process for the sagittal oblique view of the supraspinatus fossa. ${ }^{5}$ Currently, advances in ultrasonographic technology have lead to an increase in its application in musculoskeletal disorders, and the MRI Y-view can be reproduced by ultrasonography. ${ }^{4}$ However, because the Y-view is the most bony section surrounding the supraspinatus fossa, it remains challenging to obtain it using ultrasonography (Fig. 3). In this study, the sectional area of supraspinatus muscle was frequently not obtained at the section of Y-view because of the clavicle.

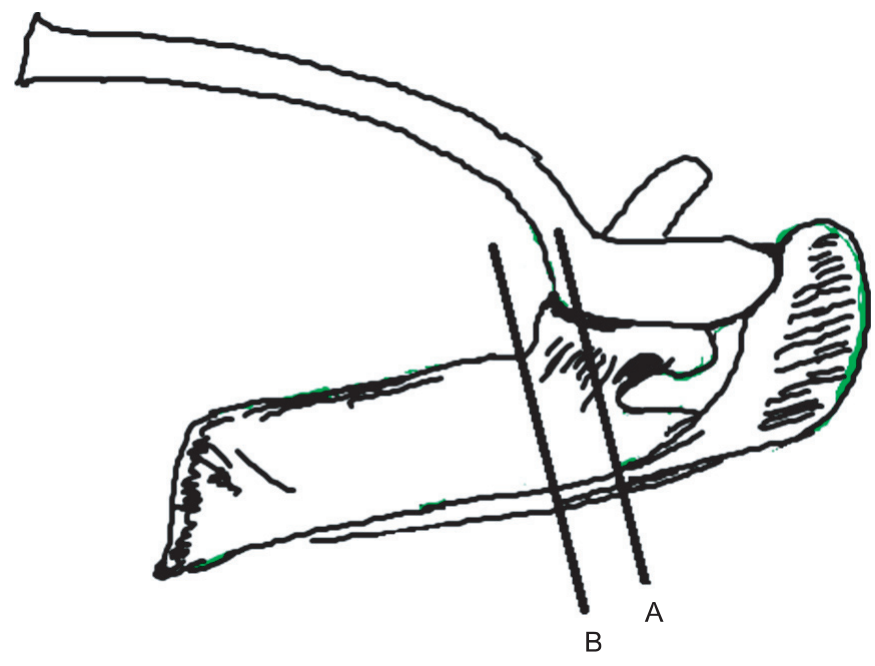

Fig. 3. (A) The sagittal oblique line on the MRI Y-view is surrounded mostly by bony boundaries. (B) The application line of the probe on ultrasonography can be displaced to avoid bony interference.

To obtain the Y-view using ultrasonography, the probe must be located longitudinal to the supraspinatus muscle, and then tilted to the deep side of the scapular spine to seek the scapular notch. After seeking the scapular notch, the probe would need to be rotated perpendicularly from the direction of the supraspinatus muscle. If the examiner can visualize the section of the supraspinatus muscle around this point, this can be considered as the Y-view (Fig. 3). If the examiner cannot visualize the entire supraspinatus muscle, the probe will need to be moved medially or laterally to obtain the entire supraspinatus muscle. Echogenecity of the bone must be observed anteriorly, inferiorly and posteriorly. The examiner should observe the clavicle anteriorly, the suprascapular fossa inferiorly and scapular spine posteriorly. Only one or two Y-views can be obtained on MRI because excessive lateral view contains the spinoglenoid notch and excessive medial view contains the scapular notch anteriorly. However, because the clavicle may overlap the supraspinatus muscle anterosuperiorly at this point, the probe may be moved a small amount medially (Fig. 3 ). In other words, Thomazeau's method could not trace the supraspinatus muscle because of the overlap of the supraspinatus muscle and the clavicle anterosuperiorly. That may be the reason why Thomazeau used the ellipse tool method in place of the tracing method. However, if the examiner moves the probe medially to avoid 
overlapping the clavicle and to locate the most lateral side of the whole section of supraspinatus, it is possible to trace the section of supraspinatus muscle. The most accurate way to measure the muscle's atrophy is by measuring the volume of the muscle. Continuative cross sectioning of the bone can mathematically calculate the volume. ${ }^{7,8}$ Nevertheless, some reports suggest only one cross sectional area for the measurement of atrophy. This method also appears to have clinical usefulness. There are reports that suggest measuring the width of the muscle correlates with the cross sectional area. ${ }^{9}$ Ultrasonography measures the cross sectional area accurately. As it is simpler to measure the thickest part of the cross section, it will be interesting to further investigate the clinical usefulness of measuring muscle thickness.

The limitations of this study are as follows. First, reliability between examiners was not measured. Second, women and elderly subjects were not evaluated in this study. In women or elderly subjects, muscular atrophy is common, and degenerated muscle is indistinguishable from fat tissue. Therefore, it remains challenging to confirm the border of the muscle in these populations. In addition, it can worsen following muscle atrophy in patients with pathologic shoulders, including shoulder pain and stroke.

\section{CONCLUSION}

In this study, the occupational ratio of the supraspinatus muscle was evaluated using ultrasonography in twenty two shoulders of eleven normal male adults. This method had low test-retest reliability, but maximum deviation was not high. Thus further studies are needed targeting patients with supraspinatus muscle atrophy due to rotator-cuff tear or stroke.

\section{REFERENCES}

1. Sheffler LR, Chae J. Neuromuscular electrical stimulation in neurorehabilitation. Muscle Nerve 2007; 35: 562-590

2. Thomazeau H, Boukobza E, Morcet N, Chaperon J, Langlais F. Prediction of rotator cuff repair results by magnetic resonance imaging. Clin Orthop Relat Res 1997; 344: 275-283

3. Lehtinen JT, Tingart MJ, Apreleva M, Zurakowski D, Palmer W, Warner JJ. Practical assessment of rotator cuff muscle volumes using shoulder of rotator cuff msucle volumes using shoulder MRI. Acta Orthop Scand 2003; 74: 722-729

4. Khoury V, Cardinal E, Brassard P. Atrophy and fatty infiltration of the supraspinatus muscle: sonography versus MRI. Am J Roentgenol 2008; 190: 1105-1111

5. Thomazeau H, Rolland Y, Lucas C, Duval JM, Langlais F. Atrophy of the supraspinatus belly, Assessment by MRI in 55 patients with rotator cuff pathology. Acta Orthop Scand 1996; 67: 264-268

6. Gottdiener JS, Livengood SV, Meyer PS, Chase GA. Should echocardiography be performed to assess effects of antihypertensive therapy? Test-retest reliability of echocardiography for measurement of left ventricular mass and function. J Am Coll Cardiol 1995; 25: $424-430$

7. Esformes JI, Narici MV, Maganaris CN. Measurement of human muscle volume using ultrasonography. Eur J Appl Physiol 2002; 87: 90-92

8. Reeves ND, Maganaris CN, Narici MV. Ultrasonographic assessment of human skeletal muscle size. Eur J Appl Physiol 2004; 91: 116-118

9. Juul-Kristensen B, Bojsen-Moller F, Holst E, Ekdahl C. Comparison of muscle sizes and moment arms of two rotator cuff muscles measured by ultrsonography and magnetic resonance imaging. Eur J Ultrasound 2000; 11: 161-173 